This is an electronic reprint of the original article. This reprint may differ from the original in pagination and typographic detail.

\author{
Author(s): Brabant, Olivier; Solati, Safa; Letule, Nerdinga; Liarmakopoulou, Ourania; Erkkilä, \\ Jaakko
}

Title: $\quad$ Favouring emotional processing in improvisational music therapy through resonance frequency breathing: a single-case experimental study with a healthy client

Year: $\quad 2017$

Version:

Please cite the original version:

Brabant, O., Solati, S., Letule, N., Liarmakopoulou, O., \& Erkkilä, J. (2017). Favouring emotional processing in improvisational music therapy through resonance frequency breathing: a single-case experimental study with a healthy client. Nordic Journal of Music Therapy, 26(5), 453-472. https://doi.org/10.1080/08098131.2016.1277253

All material supplied via JYX is protected by copyright and other intellectual property rights, and duplication or sale of all or part of any of the repository collections is not permitted, except that material may be duplicated by you for your research use or educational purposes in electronic or print form. You must obtain permission for any other use. Electronic or print copies may not be offered, whether for sale or otherwise to anyone who is not an authorised user. 


\title{
Favouring emotional processing in improvisational music therapy through resonance frequency breathing: a single-case experimental study with a healthy client
}

\author{
Olivier Brabant, Safa Solati, Nerdinga Letulè, Ourania Liarmakopoulou and Jaakko Erkkilä \\ Department of Music, Faculty of Humanities, University of Jyväskylä, Finland
}

\begin{abstract}
:
Resonance frequency breathing (RFB) is a form of slow breathing at around six breaths/min, whose immediate effects are to substantially increase heart rate variability (HRV) and to reduce stress levels. Since RFB has already been successfully used on its own to treat various emotional disorders, we wanted to evaluate its effect on emotional processing when used as a preparatory intervention in improvisational music therapy. To do so, we performed a single-subject experimental study with a healthy participant. We hypothesised that RFB would serve both as an emotional catalyst and emotional regulator, the actual outcome depending on the client's current issues and needs. The study consisted of 10 music therapy sessions, with the breathing intervention used at the beginning of every other session, in alternation with a control intervention. The data collection focussed on HRV during talking and music-making, emotion and abstraction levels in verbal content, body language, and a set of music features extracted from the client's improvisations. Our results show that the sessions starting with RFB were characterised by higher stress levels and the expression of more negative emotions, without it leading to hyperarousal and integration problems.
\end{abstract}

\section{Keywords:}

Improvisational music therapy, resonance frequency breathing, heart rate variability, stress, emotions 


\section{Background}

Since the introduction of modern Psychotherapy Process Research (PPR) in the 1950s, a wealth of reliable evidence has accumulated with the aim of answering the fundamental question "How does (psycho)therapy work?” In the process of doing so, numerous important treatment aspects have been identified and weighed in terms of their contribution to therapeutic change. Some of the most consistent aspects across theoretical orientations appear to be the role of the therapist (therapist effects), the quality of the therapeutic alliance, and the presence of emotional arousal and processing (McAleavey \& Castonguay, 2015).

In the present study, the focus is on emotional processing. Although several theories and definitions have been proposed over the years (Auszra, Greenberg, \& Herrmann, 2013), we will apply the ideas developed by Greenberg and Pascual-Leone (2006), and define emotional processing as the following three-step process. First, there needs to be emotional arousal and activation. Second, the client must be able to acknowledge, allow, and tolerate these emotions. This happens through emotional regulation, which is about finding the middle ground between avoidance and overengagement with emotions (Sloan \& Kring, 2007). Lastly, the emotional experience has to be explored and reflected upon, for example, through symbolisation and meaning-making. This definition would apply to any form of emotion-focused, experiential therapy, which includes music therapy (Pellitteri, 2009).

When looking at the literature, one recurrent finding is that emotional processing plays a key role in achieving a positive therapeutic outcome. The general idea is that more emotional processing leads to better outcomes, the opposite being true in the case of high avoidance levels (Hayes, Beevers, Feldman, Laurenceau, \& Perlman, 2005; Pos, Greenberg, \& Warwar, 2009). In other words, psychotherapy is more likely to be successful if clients express their feelings and are able to face their issues, which Hunt (1998) aptly summarised by saying "the only way out is through." Consequently, any adjunct intervention able to favour emotional processing should be considered a useful addition to the main therapy method.

The idea of preparing the client for more productive work is not new, as evidenced by the widespread use in psychotherapy of various mental imagery and relaxation techniques (Pagnini, Manzoni, Castelnuovo, \& Molinari, 2013; Singer, 1974). The use of preliminary relaxation is also the norm in certain music therapy methods, for example in Guided Imagery and Music (GIM), where every session starts with a visualisation/relaxation exercise aimed at inducing a slightly altered state of consciousness (ASC). In GIM, the induction of an ASC is considered a prerequisite for successfully journeying through the music and exploring the deeper realms of our psyche (Bruscia \& Grocke, 2002). However, with the exception of GIM, most music therapy methods do not systematically include a specific preparatory intervention at the beginning of each session.

Our interest in ASCs and their potential therapeutic benefits led us to investigate existing induction methods, with the idea of finding one intervention that could be used in improvisational music therapy, similarly to what is being done in GIM. We were looking for something safe, short, effective, affordable, and easily implementable by any therapist. The range of procedures developed throughout human history is quite wide: we find for example the ingestion of psychoactive plants, fasting, sensory deprivation, overstimulation, special breathing techniques, dancing, and drumming, 
used either separately or in combination (Winkelman, 2010). However, many of these methods were not suitable, because they would either be considered dangerous or unethical, take too much time, or be difficult to fit into a Western cultural context.

As a result, we decided to focus on breath-based interventions, since breathing is a universal phenomenon whose targeted manipulation has a long history of use as a therapeutic tool. One of the oldest example would be the Indian practice of pranayama yoga and its well-documented effect on autonomic functioning (Jerath, Edry, Barnes, \& Jerath, 2006; Pal, Velkumary, \& Madanmohan, 2004). But breathing techniques have also become increasingly popular in the West, as can be seen by their growing use for the treatment of various emotional and stress-related disorders (Brown, Gerbarg, \& Muench, 2013). Furthermore, music therapists are already familiar with certain breathing interventions, meaning that the introduction of a new method should not pose any conceptual or practical problem.

The existing breathing interventions used in music therapy can be classified into three main types. The first type consists in giving verbal instructions for breath modification as part of a relaxation induction, accompanied or followed by music listening. In this category, we find for example Progressive Muscle Relaxation (PMR), countdown inductions, and autogenic-type inductions (Grocke \& Wigram, 2007). The second type consists in giving explicit breathing cues through live or recorded music, generally with the aim of helping clients achieve slower and deeper breathing. This has been done for example in the context of burn care, with the therapist using musicreinforced relaxation to facilitate PMR and music-based imagery (Prensner, Yowler, Smith, Steele, \& Fratianne, 2001). The third type is indirect and includes all the interventions based on singing. Indeed, deeper and slower breathing naturally happens whenever we sing. This principle has been developed into specific vocal holding techniques that can be used in music psychotherapy to overcome for example early traumas or attachment issues (Austin, 2001).

Our search for a suitable intervention eventually led us to a form of slow and regular breathing, borrowed from a method called Heart Rate Variability Biofeedback (HRVB). HRVB is relying on the fact that we can directly influence our heart rate through our breathing pattern, which will consequently affect our emotional state and level of arousal. Research into cardiorespiratory coupling has demonstrated that for each person, there exists an optimal breathing speed where the amplitude of heart rate oscillations is maximised, typically at around 6 breaths per minute (Vaschillo, Lehrer, Rishe, \& Konstantinov, 2002). This maximisation of Heart Rate Variability (HRV) is accompanied by a shift of the autonomic nervous system towards parasympathetic (restand-digest) dominance, resulting in increased levels of calmness and relaxation.

Additionally, when people breathe at their optimal speed, they achieve a state called physiological coherence, where heart, respiratory, and blood pressure rhythms become highly synchronised (Lehrer \& Gevirtz, 2014). Because of the resulting amplification effect and synchronisation between several physiological systems, this optimal frequency has been dubbed the "resonance frequency.” Thus, we will henceforth refer to this type of breathing as Resonance Frequency Breathing (RFB).

In terms of applications, RFB possesses a wide range of benefits. When used on its own, RFB has been shown to enhance creativity and artistic skills (Raymond, Sajid, Parkinson, \& Gruzelier, 2005), reduce stress (Sutarto, Wahab, \& Zin, 2012), and increase people’s ability for emotional regulation 
(McCraty \& Zayas, 2014). It has also been successfully applied in the treatment of various physical and emotional disorders, such as asthma, hypertension, irritable bowel syndrome, anxiety disorders, and depression (for an overview, see Gevirtz, 2013). One specific feature of RFB is that it leads to calm alertness, a state where the person feels both relaxed and energised. This is unlike other relaxation techniques, where relaxation might be accompanied by increased sleepiness (J. C. Smith et al., 2000). Surprisingly, RFB has rarely been used in combination with other therapies, despite its ease of use, simplicity, inexpensiveness, and proven efficacy.

In the light of all these facts, we concluded that RFB would constitute a very suitable complement to improvisational music therapy. Indeed, one of the core characteristics of music improvisations is to facilitate the expression of difficult or repressed emotions by bypassing the need for verbal communication (MacDonald \& Wilson, 2014). We, therefore, hypothesised that the addition of RFB would support and enhance the benefits that clients already derive from improvisational music therapy, for example by helping them regulate the difficult emotions that might arise during improvisations. This, in turn, should have a measurable effect on HRV, verbal content, and musical expression.

More specifically, in terms of emotional processing, we postulated that RFB would have two distinct and opposite effects: it would ease the already existing negative emotions (leading to less arousal), and also favour the emergence of new or repressed emotions (leading to more arousal). What ultimately happens in the client would then depend on the nature of the client's issues. According to this hypothesis, someone in a permanent state of hyperarousal (e.g. because of anxiety disorder or post-traumatic stress disorder) would benefit mainly in terms of stress reduction. On the other hand, someone with a higher tolerance threshold would be more likely to experience difficult emotions that are normally not expressed.

This double-effect hypothesis is very relevant for music therapy in general, since music therapists are not only dealing with clients presenting emotional disorders, but also with healthy clients interested in self-development and self-actualisation (Ahonen \& Houde, 2009). It would, therefore, be important to apply RFB to healthy and unhealthy clients alike, in order to test whether the effects of RFB indeed depend on the person's current needs and emotional situation. In the absence of any prior study on RFB combined with improvisational music therapy, we decided to first use the method with healthy clients, before applying it to clients diagnosed with specific disorders.

On a more general level, studying the effect of adjunct methods is justified by the fact that music therapists anyway have to choose a session opening method, such as relaxation or an initial discussion for example. Since they are an integral part of the therapy, these opening methods have an influence on the overall effectiveness of music therapy. However, the impact of session structure is something that is not much researched in music therapy. We believe it is important to investigate the role of session structure and its various elements, in order to fine-tune the whole "package." Having a good and suitable session opening might be especially crucial in short therapy processes, where the time spent in the working phase is limited and would thus benefit from being optimised. 


\section{Method}

\subsection{Study design}

The goal of our study was to compare the effect of an experimental intervention (RFB) with the effect of a control intervention, using an alternating treatments design. The participant was a 26year-old female student with no formally diagnosed mental health issue. The control intervention consisted in sitting still while listening to relaxation music. During that time, the client was allowed to breathe freely; the only instruction we gave her was to mentally count her exhales in groups of four. RFB was used every other session, in alternation with the control intervention, so that the client would be compared to herself.

The whole process comprised 10 weekly sessions, every session starting with either 10 min of RFB or $10 \mathrm{~min}$ of relaxation, followed by $45 \mathrm{~min}$ of music therapy. Odd-numbered sessions started with RFB (i.e. session 1, 3, 5, 7, and 9), whereas even-numbered sessions (2, 4, 6, 8, and 10) started with relaxation. During the entire length of the sessions, the client was wearing a heart rate monitor (for more information, see below). The sessions were filmed with non-intrusive audiovisual equipment. Written consent was obtained from the client regarding the filming of the sessions, and the fact that the collected material would be used for teaching and/or research purposes.

\subsection{Resonance frequency breathing (RFB)}

In order to keep the experimental intervention as straightforward and uncomplicated as possible, we decided not to implement the whole HRVB procedure. This would have required that the client be fed back data in real time about his/her heart and respiration rate while performing RFB. Instead, we relied on the fact that the resonance frequency of an adult remains largely stable, the main influencing factors being gender and body height (Lehrer \& Gevirtz, 2014). This allowed us to determine the resonance frequency only once at the beginning, and then use a simple breath pacer to cue the client during the sessions starting with RFB.

The client's resonance frequency was determined by using the iteration protocol described by Lehrer (2007, Chapter 10), whereby six different breathing speeds in the neighbourhood of 6 breaths/min were consecutively tested (7, 6.5, 6, 5.5, 5, and 4.5 breaths/min). After 4 min of data collection at each of these speeds, we computed and compared the HRV power spectra of each measurement, based on the last 3 min of data (for the detailed HRV methodology, see below). The optimal speed was defined as the speed that yielded the highest spectral peak in the vicinity of 0.1 $\mathrm{Hz}$. In this specific case, the client's resonance frequency was 6.5 breaths/min, corresponding to a spectral peak at $0.108 \mathrm{~Hz}$.

When using RFB, another important aspect to consider besides the breathing speed is the inhalation/exhalation (i/e) ratio. Generally speaking, we know that HRV is higher when exhalations last longer than inhalations (Strauss-Blasche et al., 2000). Furthermore, in a study investigating specifically the effect of the i/e ratio when breathing at 6 breaths/min (Diest et al., 2014), participants reported more relaxation, positive energy, stress reduction, and mindfulness with a low i/e ratio (exhales $>$ inhales), compared to a high i/e ratio (inhales $>$ exhales). In the present study, the i/e ratio was set to $40 / 60$, meaning that inhalation lasted $3.7 \mathrm{~s}$ and exhalation $5.5 \mathrm{~s}$. 
Both the resonance frequency assessment and the RFB intervention were performed in the same way. Every time, the client was seated in front of a screen and asked to follow the auditory and visual cues of a respiratory pacing programme. The client was given the following breathing instructions: to use abdominal breathing, to breathe in through the nose and out through the mouth with pursed lips, and to keep the breathing shallow and natural so as to avoid hyperventilation. The breathing intervention was well-received by the client and did have the expected maximisation effect on HRV (see the Results). However, despite the difference in physiological response between RFB and relaxation, it should be noted that the client did not report any difference in terms of perceived relaxation effect.

\subsection{Music therapy}

Regarding the music therapy part, the chosen model was Integrative Improvisational Music Therapy (IIMT), which is a model that was developed at the University of Jyväskylä, Finland, and successfully used for the treatment of depression in a randomised controlled trial (Erkkilä et al., 2011). In IIMT, clients are encouraged to express themselves musically in order to explore their thoughts, memories, emotions, and inner conflicts. The therapist is playing together with the client, thus creating a shared musical experience. A typical session consists of two alternating phases: an improvisation phase - with its focus on imagery, associations, and symbolism - and a verbal phase where themes are discussed and reflected upon (Erkkilä, Punkanen, \& Fachner, 2012).

The music improvisations were performed either on a djembé drum or on a malletKAT Pro. The malletKAT Pro is a MIDI controller that has the same key layout as a marimba, and is played using one or two mallets. In this specific study, it was set to emulate the sound of a vibraphone. When improvising together, client and therapist were facing each other and playing the same type of instrument. Both the djembés and malletKATs were connected to a computer running Logic Pro (version 10.2), which enabled us to record the improvisations in the form of audio data (from the djembés) and MIDI data (from the malletKATs).

\section{Data collection and analysis}

Regarding the data collection and its subsequent analysis, we opted for a multi-method quantitative approach, whereby the music therapy sessions were analysed from four different angles: HRV, session transcripts, video material, and music features. The methodology was quantitative in the sense that each data source produced numerical data that would then be analysed statistically and compared. This type of data triangulation is a powerful methodology, because it enables the researchers to cross-validate and strengthen the findings obtained from each individual data source (Flick, 2004). HRV data for example would be more difficult to interpret without any information about the corresponding real-life events. Similarly, observational or descriptive data can greatly benefit from the addition of objective information such as physiological data.

\subsection{Heart rate variability (HRV)}

\subsubsection{Data acquisition and pre-processing}

Heart rate data were acquired using a Suunto Memory Belt, which is the chest strap component of the Suunto t6 heart rate monitor. The Memory Belt has a $1000 \mathrm{~Hz}$ sampling rate (i.e. $1 \mathrm{~ms}$ accuracy), 
and it has been shown to be reliable when compared to a 5-lead electrocardiogram system (Weippert et al., 2010). Data pre-processing and analysis were performed with version 2.2 of Kubios, an HRV analysis software developed at the University of Eastern Finland (Tarvainen, Niskanen, Lipponen, Ranta-aho, \& Karjalainen, 2014).

Since data were obtained from a young healthy adult with only occasional abnormal heartbeats, we relied on Kubios' automatic detection and correction feature to remove artefacts. Kubios performs artefact correction on the basis of five sensitivity thresholds. Each threshold defines how much a beat-to-beat interval must deviate from the local average before being considered abnormal, with a range from "very low" (0.45 sec) to "very strong” ( $0.05 \mathrm{sec})$. Following detection, unwanted heartbeats are automatically replaced using a cubic spline interpolation method. After examining the data for ectopic beats and other artefacts, we selected the minimum sensitivity level needed to get rid of the artefacts without affecting the rest of the data.

Furthermore, before performing the HRV analysis, long-term trends corresponding to very slow fluctuations were systematically removed using the smoothness priors approach, with a $\lambda$ value of 500 (Tarvainen, Ranta-aho, \& Karjalainen, 2002). This approach is roughly equivalent to applying a high-pass filter, where the chosen $\lambda$ value determines the cut-off frequency (in this case, $500 \approx 0.035 \mathrm{~Hz}$ ). Detrending has two benefits: it removes unwanted information from the data and renders the heart rate signal more stationary, which is a prerequisite for accurately performing a power spectral analysis (Magagnin et al., 2011).

\subsubsection{Chosen metrics}

In the time domain, we calculated the standard deviation of all N-N (beat-to-beat) intervals (SDNN), and the root mean square differences of successive N-N intervals (RMSSD). SDNN is a measure of overall HRV variance, whereas RMSSD represents the amount of short-term variability.

In the frequency domain, we performed a power spectral analysis using the Fast Fourier Transform (FFT) algorithm, with a data interpolation rate of $4 \mathrm{~Hz}$. We thus obtained power values for the following frequency bands: very low frequency (VLF, 0-0.04 Hz), low frequency (LF, $0.04-0.15$ $\mathrm{Hz}$ ), and high frequency (HF, $0.15-0.4 \mathrm{~Hz}$ ). However, since the inclusion of VLF in the analysis of short-term measurements is problematic (Task Force, 1996), we only focused on LF and HF. Because the results produced by a power spectral analysis are typically not normally distributed, we applied a logarithmic transformation (natural log, ln) to the absolute values of LF and HF. We also converted those absolute values to normalised units (nu) in order to obtain the relative power distribution between LF and HF (in proportion to the total power minus the VLF component).

Under normal circumstances, HF is a marker of vagal tone and parasympathetic activity (Task Force, 1996; Thayer, Yamamoto, \& Brosschot, 2010), with high HF indicating that the parasympathetic branch of the autonomic nervous system is dominant (low physiological arousal). There is, for example, strong evidence suggesting that the practice of meditation results in an increase of (relative) HF power (An, Kulkarni, Nagarathna, \& Nagendra, 2010; Krygier et al., 2013; Wu \& Lo, 2008). Conversely, low HF would typically be the result of stress and anxiety (Cohen et al., 1998; Schwarz, Schächinger, Adler, \& Goetz, 2003).

Contrary to HF, LF is rather difficult to interpret. Indeed, it has now been established that LF is not an index of pure sympathetic activity, but a mix of sympathetic, parasympathetic, and baroreflex 
influences (Shaffer, McCraty, \& Zerr, 2014). This interpretation issue makes it problematic to use LF/HF as an index of sympathovagal balance (Reyes del Paso, Langewitz, Mulder, van Roon, \& Duschek, 2013), which is why we decided not to report this ratio, although it is still widely used in the literature. Besides, LF/HF is anyway redundant information with LFnu and HFnu, these three measures being mathematically equivalent and displaying a curvilinear relationship (Heathers, 2014).

\subsubsection{Segmentation and averaging}

Conventional HRV studies usually take place in a very controlled environment, with all the participants performing the exact same task during a predefined amount of time. Typically, if the researchers are interested in short-term HRV, they will use 5-minute data segments, in accordance with the recommendations of the Task Force of the European Society of Cardiology and the North American Society of Pacing (1996).

However, such an approach is not suitable for IIMT. Indeed, music improvisations are by definition unique and spontaneous creations that can last any amount of time, from a few minutes to the entire length of the session. The same applies to the moments of verbal exchange. In order to adequately capture all the moments of interest occurring in a therapy session, we needed a methodology flexible enough to be applied to data segments of varying length, while providing enough precision and reliability.

First of all, recent studies have demonstrated that many of the commonly-used HRV metrics remain reliable in short-term measurements lasting less than 5 min (Salahuddin, Cho, Jeong, \& Kim, 2007; A.-L. Smith, Owen, \& Reynolds, 2013). However, when performing a power spectral analysis, a minimum of $2 \mathrm{~min}$ is required for accurately capturing both the LF and HF components of heart rate oscillations (Berntson et al., 1997). Furthermore, a general rule for HRV measurements is that one should only compare data segments of equal length (Task Force, 1996). Taking all these facts into account, we decided to use exclusively 2-minute data segments in our analysis.

In order to achieve the desired flexibility, we used the approach suggested and validated by Rivecourt et al. (2008). The authors showed how the average of several overlapping 2-minute segments can be used to reliably estimate the mean spectral values of a longer data segment. Therefore, we chose a methodology based on the averaging of multiple 2-minute segments, with a segment overlap of 50\%. This enabled us to analyse therapy moments of any length-provided that they lasted at least $2 \mathrm{~min}$ - with a time resolution of $1 \mathrm{~min}$, as determined by the amount of segment overlap. SDNN and RMSSD were calculated together with the spectral values, using the same averaging method.

\subsection{Text and video materials}

The content analysis of the therapy sessions focused on the client, and took into account both the verbal and non-verbal communication occurring between client and therapist. The two data sources used in the analysis were the video recordings and transcripts of the therapy sessions. Although these typically constitute the source material of qualitative research, in this study we opted for a quantitative approach, in order to allow proper juxtaposition and comparison with the other (purely numerical) data sources. 
Verbal interactions were analysed with the help of the CM software (version 4.3), a computer-based text analysis tool developed by Mergenthaler (1996, 2008). This automated tool was designed to identify emotion-abstraction patterns in session transcripts, in accordance with the Therapy Cycle Model (TCM; see Mergenthaler, 1996). The TCM and related software are school-independent, meaning they are applicable to a wide range of psychotherapeutic approaches, provided there is sufficient verbal content to be analysed.

To perform the analysis, CM relies on two thematic dictionaries ${ }^{1}$, one for affective language (emotion words) and another for conceptual language (abstract words). When applied to session transcripts, it measures the density of emotion and abstract words in a given text unit, resulting in a frequency distribution for each category. Furthermore, the identified words are given a valence label: positive (A) or negative (B) for emotion words, and positive (C), negative (D), or neutral (E) for abstract words. By combining the positive and negative words of both categories, two new categories are created that reflect the valence of the overall emotional tone $(E T)$ : positive ET $(A+C)$ and negative ET $(\mathrm{B}+\mathrm{D})$.

In the present study, each therapy session was first transcribed according to the transcription standards defined by Mergenthaler and Stinson (1992). The application of these standards is required for the CM software to properly analyse the text. We then performed a corpus analysis of all the 10 sessions, each session representing one unit of analysis. The results gave us the relative frequency of each of the four categories mentioned above (Emotion, Abstraction, Positive ET, and Negative ET).

Using the video material as the data source, we also focused on one specific aspect of non-verbal communication, namely on an idiosyncratic form of smiling. Indeed, while watching the video recordings, we noticed that the client tended to exhibit a type of tense and bitter smile whenever the discussion became emotionally challenging. Consequently, we created the category Negative Smile and counted its relative occurrence in each session. The result was expressed as a proportion of the total session duration.

\subsection{Music features}

For the music analysis, we only used data extracted from the client's malletKAT improvisations. Indeed, malletKAT improvisations were performed in every session except one, whereas djembé improvisations only took place in six sessions out of ten, making them too infrequent to be included in a comparative experiment. There was a total of 11 malletKAT improvisations, the client performing usually one improvisation per session, except in sessions 1 and 8 where she performed two. All the improvisations were analysed separately. Their average length was $10 \mathrm{~min} 28 \mathrm{sec}$, the shortest improvisation lasting $3 \mathrm{~min} 20 \mathrm{sec}$ and the longest $40 \mathrm{~min} 37 \mathrm{~s}$.

In order to access the numerical data contained in the MIDI files and run descriptive statistics, we first converted each MIDI file into Comma-Separated Value (.csv) text files. For each improvisation, we then extracted the following information: the lowest note played (note_min), the highest note played (note_max), how hard each note was played (velocity), and how many notes were played (number). Velocity is a way of measuring the volume of a note, since the harder we hit a key, the

\footnotetext{
${ }^{1}$ For more information on the development of these dictionaries, see Mergenthaler (1996).
} 
louder the note. In MIDI data, pitch and velocity information is represented as a number ranging from 0 to 127 (in the case of pitch, 0 is C1 and 127 is G9, whereas for velocity, 0 is the softest possible keystroke and 127 the hardest). Regarding the amount of notes, it is based on the total number of note onsets.

The analysis itself focussed on the notes' range (note_min, note_max), the amount of total variation in pitch (note_SD), the amount of total variation in dynamics (velocity_SD), and the amount of notes played (number). Additionally, we also looked at how long the improvisations were (length).

\section{Results}

The four data sources were first analysed separately, using independent samples $t$-tests to compare the means obtained under condition 1 (RFB) and under condition 2 (relaxation). We then performed a correlation analysis to identify possible associations between the data sources. For an overview of all the metrics included in the analysis, as well as the corresponding measurement units, see Table 1.

Table 1. Measurement units

\begin{tabular}{|c|c|}
\hline \multicolumn{2}{|l|}{ HRV } \\
\hline SDNN, RMSSD & $\mathrm{ms}$ \\
\hline LF, HF & $\mathrm{ms}^{2}, \log$ transformed (natural log, In) \\
\hline LFnu & normalised units: LF / (total power - VLF) \\
\hline HFnu & normalised units: HF / (total power - VLF) \\
\hline HR & beats/min \\
\hline \multicolumn{2}{|l|}{ Content analysis } \\
\hline \multicolumn{2}{|c|}{$\begin{array}{l}\text { Emotion, Abstraction, } \\
\text { Positive ET, Negative ET }\end{array}$} \\
\hline Negative smile & $\%$ of total session duration \\
\hline \multicolumn{2}{|l|}{ Music features } \\
\hline $\begin{array}{l}\text { velocity_SD, note_SD, } \\
\text { note_min, note_max }\end{array}$ & MIDI value $(0-127)$ \\
\hline length & s \\
\hline number & number of notes \\
\hline
\end{tabular}

As can be seen from Table 2, during the initial intervention, RFB produced significantly higher levels of SDNN (overall HRV) than the relaxation intervention, which was to be expected. Additionally, there was a stronger concentration of spectral power in the LF band during RFB than during relaxation, both in absolute terms (LF) and relative terms (LFnu). This was also an expected result, since one characteristic of RFB is to produce a spectral peak around the resonance frequency $(0.108 \mathrm{~Hz})$, which is located in the LF band. As a side note, this co-occurrence of high HRV and a massive increase in LF power should be seen as an exception that only happens when performing RFB. Indeed, when breathing normally, increased relaxation would typically be accompanied by increased HF power (absolute and/or relative), since HF is the usual marker of parasympathetic activity under normal circumstances. 
Table 2. Results of t-test for HRV measures

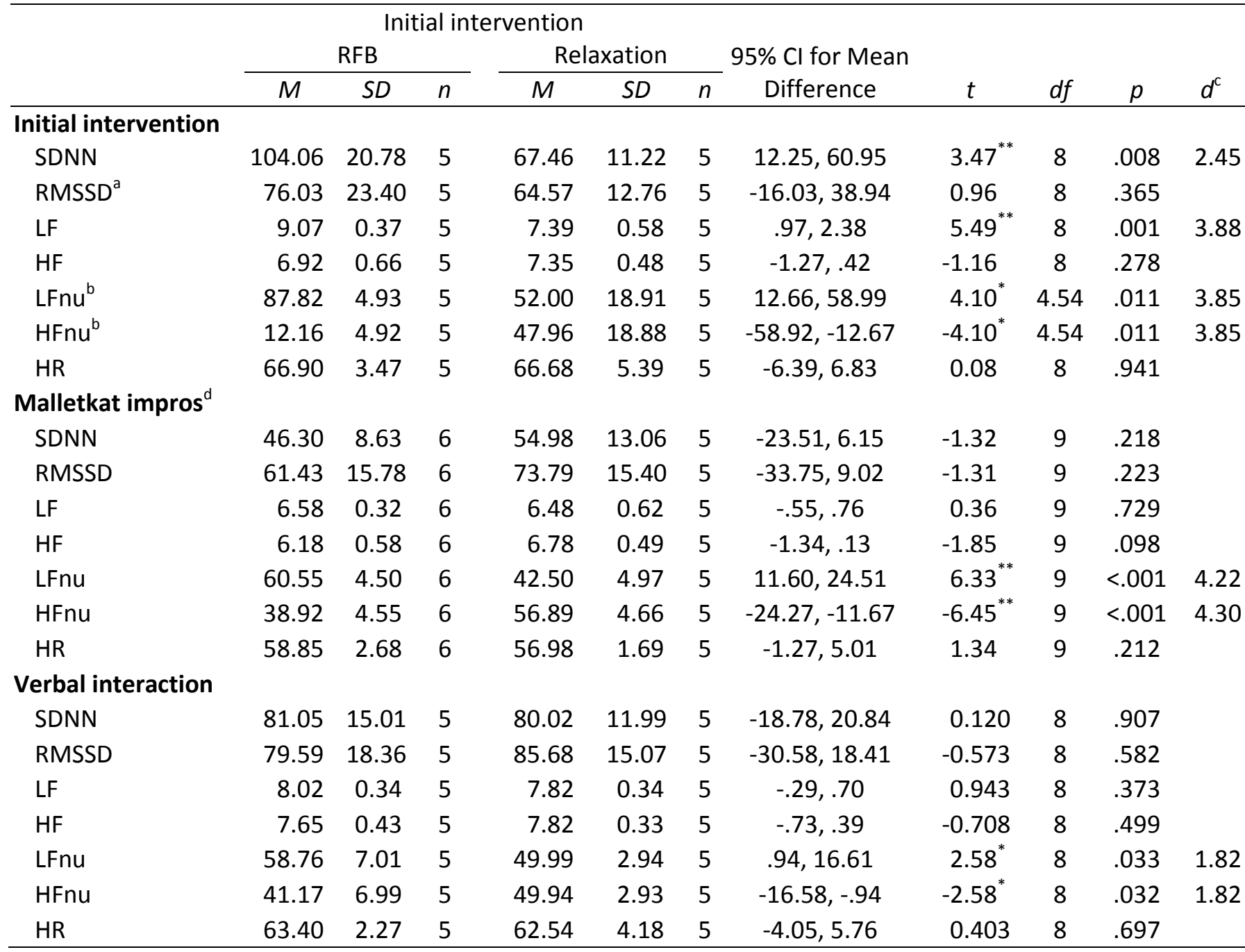

${ }^{\mathrm{a}}$ Values were not normally distributed. A Mann-Whitney $U$ test confirmed the non-significance of the result $(U=7, p=.251)$.

${ }^{\mathrm{b}}$ Equal variances not assumed (Levene's test was significant with $p<.05$ ).

${ }^{\mathrm{c}}$ Formula used to calculate the effect size (Cohen's $d$ ): $2|t| / \sqrt{ } d f$

${ }^{\mathrm{d}}$ Each malletKAT improvisation was considered separately. There was one such improvisation per session, except for session 6 (no malletKAT improvisation), and sessions 1 and 8 (two improvisations each). ${ }^{*} p<.05,{ }^{* *} p<.01$

Regarding the effects of RFB on the rest of the music therapy session, significant effects were found for LFnu and HFnu, both during verbal interaction and malletKAT improvisations (see Table 2). In both cases, RFB was followed by higher LFnu and lower HFnu, compared to sessions starting with relaxation. As indicated above, lower HFnu can be interpreted as lower parasympathetic activity. In other words, the client was apparently experiencing more stress and negative emotions during the sessions starting with RFB, and this effect was visible while talking and improvising. We found no significant difference between conditions 1 and 2 in the remaining HRV measures (see Table 2).

The idea that sessions starting with RFB were emotionally more challenging for the client was corroborated by the findings of the content analysis. Indeed, there was a statistically significant difference in Negative Smile between conditions, with more Negative Smile in sessions starting 
with RFB (see Table 3). Furthermore, when considering sessions 4-10, the amount of emotional words was systematically higher after RFB than after relaxation, and the overall emotional tone also tended to be more negative after RFB, but the latter result only approached statistical significance $(p=.062)$. Here are a few examples of the difficult topics that emerged: disappointment, frustration, panic, fear, feeling empty inside, and feeling trapped.

Table 3. Results of t-test for the content analysis (verbal and non-verbal interaction)

\begin{tabular}{|c|c|c|c|c|c|c|c|c|c|c|c|}
\hline & \multicolumn{6}{|c|}{ Initial intervention } & \multirow{3}{*}{$\begin{array}{l}\text { 95\% Cl for Mean } \\
\text { Difference }\end{array}$} & \multirow[b]{3}{*}{$t$} & \multirow[b]{3}{*}{$d f$} & \multirow[b]{3}{*}{$p$} & \multirow[b]{3}{*}{$d^{\mathrm{b}}$} \\
\hline & \multicolumn{3}{|c|}{ RFB } & \multicolumn{3}{|c|}{ Relaxation } & & & & & \\
\hline & $M$ & $S D$ & $n$ & $M$ & $S D$ & $n$ & & & & & \\
\hline \multicolumn{12}{|l|}{ All sessions } \\
\hline Emotion & 5.40 & 1.04 & 5 & 4.43 & 0.50 & 5 & $-.22,2.17$ & 1.88 & 8 & .097 & \\
\hline Abstraction & 5.47 & 1.13 & 5 & 6.33 & 1.14 & 5 & $-2.51, .79$ & -1.20 & 8 & .264 & \\
\hline Positive ET & 3.63 & 1.01 & 5 & 3.69 & 0.71 & 5 & $-1.34,1.21$ & -0.12 & 8 & .908 & \\
\hline Negative $\mathrm{ET}^{\mathrm{a}}$ & 2.26 & 1.02 & 5 & 1.30 & 0.35 & 5 & $-.29,2.21$ & 1.98 & 4.94 & .106 & \\
\hline Negative Smile & 1.86 & 0.78 & 5 & 0.68 & 0.57 & 5 & $.17,2.18$ & $2.70^{*}$ & 8 & .027 & 1.91 \\
\hline \multicolumn{12}{|l|}{ Sessions 4 to 10} \\
\hline Emotion & 5.84 & 0.80 & 3 & 4.45 & 0.58 & 4 & $.07,2.72$ & $2.70^{*}$ & 5 & .043 & 2.42 \\
\hline Abstraction & 6.18 & 0.81 & 3 & 6.15 & 1.22 & 4 & $-2.08,2.14$ & 0.04 & 5 & .971 & \\
\hline Positive ET & 3.78 & 1.37 & 3 & 3.49 & 0.63 & 4 & $-1.65,2.24$ & 0.39 & 5 & .712 & \\
\hline Negative ET & 2.65 & 1.01 & 3 & 1.41 & 0.31 & 4 & $-.09,2.58$ & 2.39 & 5 & .062 & \\
\hline Negative Smile & 2.23 & 0.84 & 3 & 0.53 & 0.54 & 4 & $.38,3.02$ & $3.31^{*}$ & 5 & .021 & 2.96 \\
\hline
\end{tabular}

Interestingly enough, Positive ET did not display any significant difference between conditions, which means that positive and negative emotional tone should be seen as two independent dimensions, rather than two ends of the same spectrum. This result is in line with findings from mood research, where negative affect and positive affect have been found to be two largely independent dimensions (Watson, Clark, \& Tellegen, 1988). As to the presence of conceptual language, there was no difference either between RFB and relaxation. Instead, starting from session 3 , we observed a steady increase of abstract content, independently of the initial intervention (see Figure 1).

In terms of correlations involving the content analysis and HRV measures during verbal interaction, we found a negative relationship between Emotion and HFnu ( $r=-0.64, p=.048, n=10)$, indicating that higher emotional content was accompanied by higher levels of stress. No further correlations were found between the remaining measures of verbal/non-verbal content and HRV 


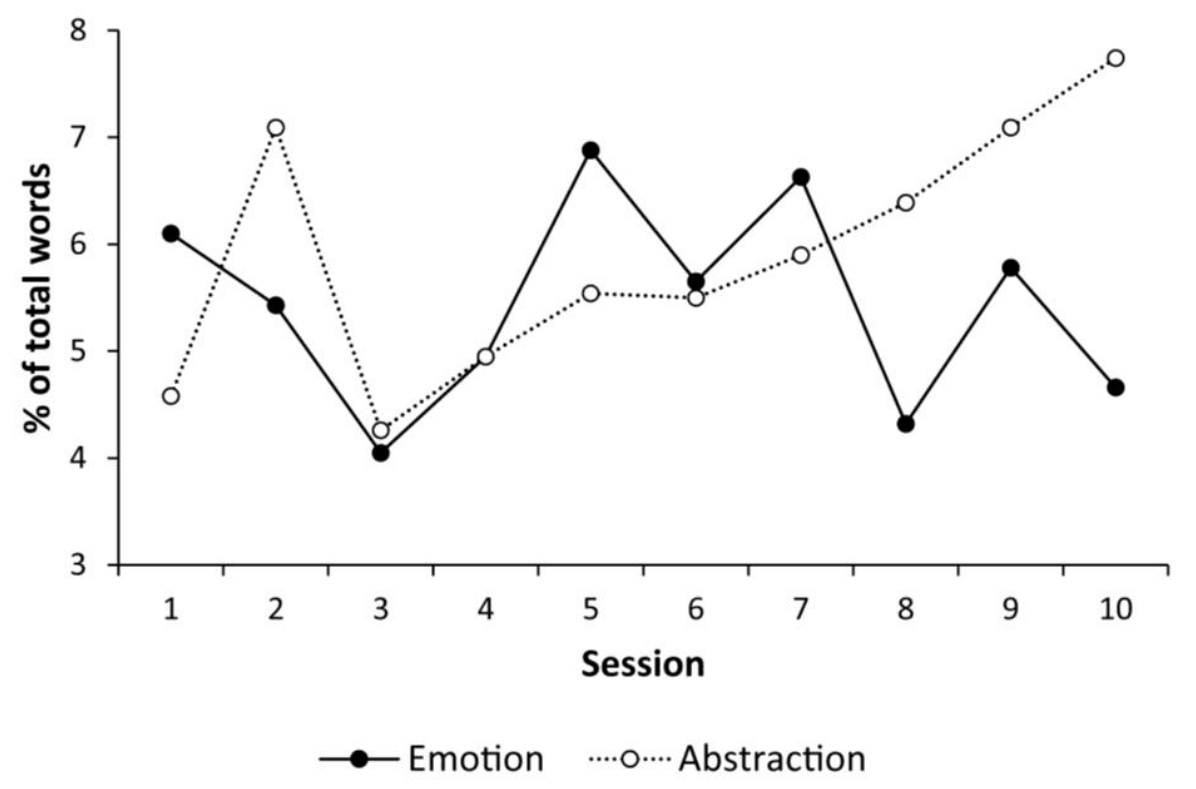

Figure 1. Emotional and abstract content in the client's verbal expression.

during verbal interaction. However, we found a positive correlation between Negative Smile and Negative ET ( $r=0.68, p=.03, n=10$ ), which became especially strong in the second half of the therapy process ( $r=0.97, p=.007, n=5$; see Figure 2 ). This finding strengthens our hypothesis that the non-verbal event we called Negative Smile is indeed linked to the expression of negative emotions in this particular client.

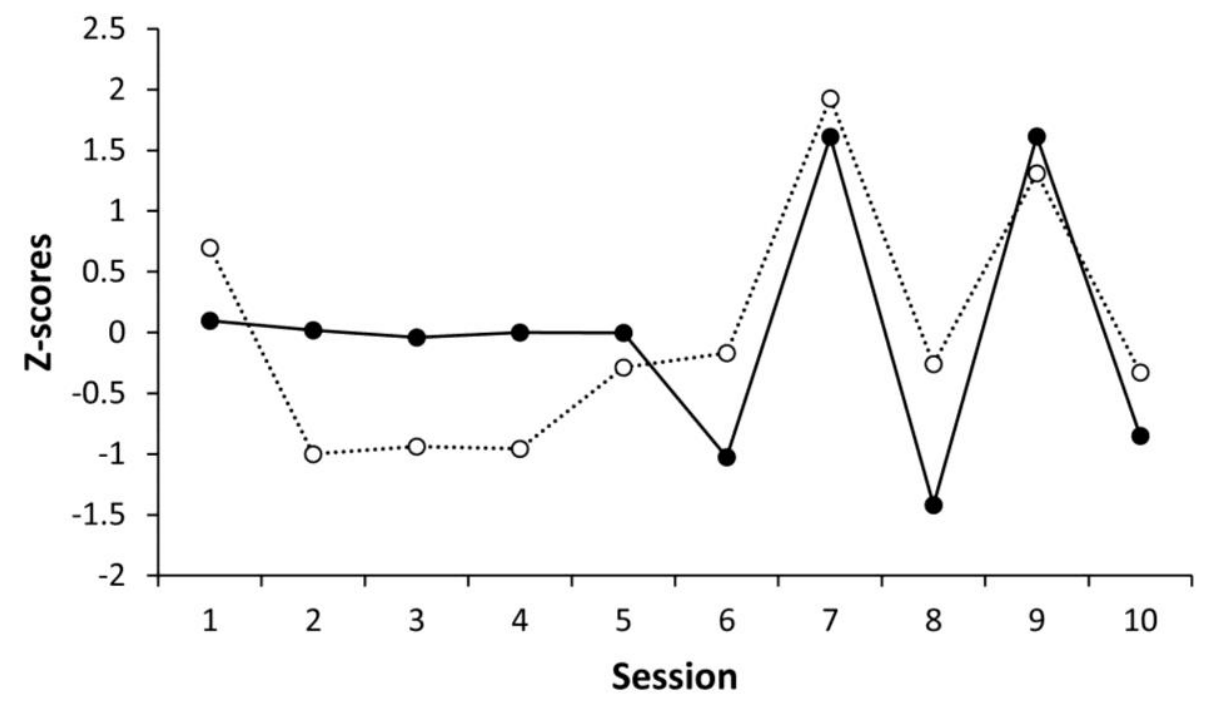

$\longrightarrow$ Negative Smile $\quad \cdots . . . \cdots$ Negative ET

Figure 2. Comparison between the amount of negative smile and negative emotional tone.

Turning now our attention to the music features of the malletKAT improvisations, no music feature displayed any statistically significant difference between conditions 1 and 2 . There was, however, one noteworthy correlation involving velocity_SD and HRV during improvisations: velocity_SD 
correlated negatively with HFnu, meaning that increased stress levels (low HFnu) were accompanied by more variation in dynamics (i.e. volume levels were less constant). The detailed results of this correlation analysis can be found in Table 4 .

Table 4. Correlation coefficients between music features (malletKAT) and HRV (malletKAT)

\begin{tabular}{lcccccccc}
\hline & \multicolumn{7}{c}{ HRV measures } \\
\cline { 2 - 9 } & & SDNN & RMSSD & LF (In) & HF (In) & LFnu & HFnu & HR \\
\hline Music features & & & & & & & \\
velocity_SD & $r$ & -.36 & -.41 & .16 & -.32 & $.62^{*}$ & $-.61^{*}$ & .59 \\
note_SD & $r$ & .12 & .00 & .52 & .20 & .37 & -.37 & .24 \\
note_min & $r$ & .17 & .15 & -.46 & -.11 & -.42 & .41 & -.40 \\
note_max & $r_{s}$ & .15 & .32 & .50 & .02 & .24 & -.24 & .16 \\
length & $r_{s}$ & -.01 & .05 & .50 & .14 & .04 & -.04 & .20 \\
number & $r_{S}$ & -.10 & .06 & .43 & -.05 & .34 & -.34 & .35 \\
\hline
\end{tabular}

$n=11$ for all analyses.

Each malletKAT improvisation was considered separately ${ }^{*} p<.05$

Table 5. Correlation coefficients between music features (malletKAT) and verbal/non-verbal content

\begin{tabular}{|c|c|c|c|c|c|c|}
\hline & & \multicolumn{5}{|c|}{ Content analysis } \\
\hline & & Emotion & Abstraction & Positive ET & Negative ET & Negative Smile \\
\hline \multicolumn{7}{|l|}{ Music features } \\
\hline velocity_SD & $r$ & .57 & -.57 & .43 & .01 & .20 \\
\hline note_SD & $r$ & $.74^{*}$ & -.51 & $.76^{*}$ & .03 & .38 \\
\hline note_min & $r$ & -.47 & .45 & $-.80^{* \star}$ & .25 & -.23 \\
\hline note_max & $r_{s}$ & .52 & -.63 & $.68^{*}$ & -.27 & .36 \\
\hline length & $r_{s}$ & .28 & -.24 & $.83^{* *}$ & $-.67^{\dagger}$ & -.12 \\
\hline number & $r_{s}$ & $.70^{*}$ & -.59 & $.72^{*}$ & -.10 & .22 \\
\hline
\end{tabular}

$n=9$ for all analyses.

Session averages were used for the malletKAT improvisations.

${ }^{*} p<.05,{ }^{* *} p<.01,{ }^{\dagger} p=.05$

Furthermore, we found several significant correlations between the music features of the malletKAT improvisations played in a given session and the emotional content of the verbal exchange that took place in that same session. As shown in Table 5, when emotional content increased, the client played more notes and used a wider range of notes. When Positive ET increased, the client played longer, used more notes and a wider range of notes, and both the lower and higher register expanded. Lastly, when Negative ET increased, the client played shorter improvisations.

\section{Discussion}

The purpose of this study was to investigate the effects of resonance frequency breathing (RFB) on autonomic functioning, emotional processing, and musical expression during improvisational music therapy. Because of the ability of RFB to dramatically increase HRV in the short term, and given its successful implementation in the treatment of various emotional disorders, we hypothesised that the 
addition of RFB would be beneficial to the therapy process.

The results showed that RFB, as an initial intervention, did have an effect that distinguished it from relaxation, both immediately and during the music therapy session that followed. This effect was visible in the HRV data, as well as in the verbal and non-verbal content. As expected, RFB increased relaxation levels while it was being performed, with higher HRV during RFB compared to music listening. However, during the rest of the session, RFB was followed by reduced parasympathetic activity (more stress) while talking and improvising, more emotional content while talking, and more negative smiling. We also found a trend towards more negative emotional tone after RFB.

The fact that the client was experiencing more stress in the sessions starting with RFB might appear paradoxical, given the relaxation effect that we would expect from RFB. To solve this apparent paradox, it is important to distinguish what happened during the breathing from what happened afterwards. During the breathing itself, RFB did have the expected relaxation effect. As to the rest of the session, we should keep in mind that the participant was a healthy client with no emotional disorder. As we explained in our hypotheses, one of the effects we expected from RFB was the emergence of negative emotions, which in turn would lead to more arousal. Our triangulation approach confirmed that the increased stress was indeed connected with more difficult emotional topics. This can be considered a good thing from a therapeutic perspective, provided of course that the client was able to handle the increased stress levels. So the question is, was it a problem for the client to confront her issues and be more stressed while doing so?

Based on the results presented here, we believe the answer is no. Indeed, the fact that the client expressed and explored these emotions together with the therapist indicates that she was able to face these emotions and "stay" with them. In other words, appropriate emotional regulation did take place alongside the emotional activation. Additionally, we saw that the relative amount of conceptual language steadily increased from the third session onwards, which indicates that she was able to verbally process the negative emotions that emerged during therapy.

However, one thing we cannot know for certain is the extent to which RFB actually increased the client's window of tolerance, or whether this window remained unchanged. Indeed, it might be that the amount of negative arousal just happened to take place inside the client's usual boundaries, without there being any improvement in flexibility and tolerance. Although prior studies indicate that RFB does have a positive effect on emotional regulation (McCraty \& Zayas, 2014), in the absence of direct measurements, this last point remains an open question.

Another question that remains unanswered is the duration of the effect that can be attributed to RFB. Was the RFB intervention influencing the entire music therapy session that followed, or only a part of it? We know from existing studies that a carry-over effect does exist, at least on HRV and in the short term. For example, Karavidas et al. (2007) and Zucker et al. (2009) both reported increased SDNN during the 5-minute resting period immediately following RFB, when compared to the preintervention resting period. However, we do not know how long this beneficial effect on HRV actually lasts, and what would be the factors responsible for intra- and inter-individual variations.

Anyway, in the case of RFB used as a preparatory intervention within music therapy, the question of carry-over effect cannot be answered satisfactorily by studying only the impact on HRV at rest. 
Indeed, in our proposed model (RFB followed by IIMT), the client is not resting but actively engaged in therapy, which is arguably a rather different activity. Actually, given the characteristics and common qualities of RFB and IIMT, we would like to propose that the two methods are in fact supporting each other, with IIMT not only maintaining but also amplifying the process initiated by RFB. If such is indeed the case and a positive feedback loop is created, it would mean that the effects of RFB can be sustained for the entire length of the therapy session.

Regarding the effects of RFB on HRV, we only found significant differences between conditions in relative HRV measures (LFnu and HFnu), and none in absolute measures (SDNN, RMSSD, LF, $\mathrm{HF}$ ). This is very likely due to the fact that in the same individual, natural variations in absolute HRV measures can be quite strong from one recording to the next, typically between 10 and 30\% (Sandercock, Bromley, \& Brodie, 2005). Therefore, a true effect might not be detected if the difference in absolute HRV measures following an intervention is lower than this natural intraindividual variability. Because they are expressed in the form of ratios and proportions, relative HRV measures are obviously not affected by this problem.

From the four data sources under investigation, only the music features did not display systematic fluctuations that could be attributed to the preparatory intervention. We did find, however, a link between certain music features and HRV levels during the corresponding improvisations, as well as with the emotional valence of the verbal interaction taking place in the same session. To summarise, when positive emotional tone increased, the client used a wider range of notes and played longer improvisations. Moreover, when she was calmer, improvisations were more monotonous and stable in terms of loudness. Quite obviously, further studies with a similar set-up are required in order to find out whether these associations are generalisable beyond this specific client.

The findings of the present study are in line with the hypothesis that healthy clients will experience increased stress levels during therapy as a result of RFB, conceivably because they possess a higher tolerance threshold than clients with emotional disorders. However, in order to truly test whether the effects of RFB are adaptative and dependent on the client's needs, it would be necessary to conduct further studies with more participants, including client groups having a specific diagnosis. Indeed, although the results seem promising, it is not possible at this stage to make any strong statement or to generalise the findings to a larger population.

Lastly, besides studying the effects of RFB on therapy processes, it would also be useful to add outcome measures and investigate whether these effects are clinically relevant. If the positive effects on emotional processing are confirmed and a clear link to better therapeutic outcomes can be established, it would open the possibility for more effective and shorter therapy, as well as reduced healthcare costs.

\section{Acknowledgements}

The authors would like to thank Prof. Erhard Mergenthaler for kindly providing the text analysis software used in this study. 


\section{References}

Ahonen, H., \& Houde, M. (2009). Something in the air: Journeys of self-actualization in musical improvisation. Voices: A World Forum for Music Therapy, 9(2). https://doi.org/10.15845/voices.v9i2.348

An, H., Kulkarni, R., Nagarathna, R., \& Nagendra, H. R. (2010). Measures of heart rate variability in women following a meditation technique. International Journal of Yoga, 3(1), 6. https://doi.org/10.4103/0973-6131.66772

Austin, D. (2001). In search of the self: The use of vocal holding techniques with adults traumatized as children. Music Therapy Perspectives, 19(1), 22-30. https://doi.org/10.1093/mtp/19.1.22

Auszra, L., Greenberg, L. S., \& Herrmann, I. (2013). Client emotional productivity-optimal client in-session emotional processing in experiential therapy. Psychotherapy Research, 23(6), 732-746. https://doi.org/10.1080/10503307.2013.816882

Berntson, G. G., Thomas, J., Eckberg, D. L., Grossman, P., Kaufmann, P. G., Malik, M., ... van der Molen, M. W. (1997). Heart rate variability: Origins, methods, and interpretive caveats. Psychophysiology, 34(6), 623-648. https://doi.org/10.1111/j.1469-8986.1997.tb02140.x

Brown, R. P., Gerbarg, P. L., \& Muench, F. (2013). Breathing practices for treatment of psychiatric and stress-related medical conditions. Psychiatric Clinics of North America, 36(1), 121-140. https://doi.org/10.1016/j.psc.2013.01.001

Bruscia, K., \& Grocke, D. (2002). Guided Imagery and Music: The Bonny method and beyond. Gilsum, NH: Barcelona Publishers.

Cohen, H., Kotler, M., Matar, M. A., Kaplan, Z., Loewenthal, U., Miodownik, H., \& Cassuto, Y. (1998). Analysis of heart rate variability in posttraumatic stress disorder patients in response to a trauma-related reminder. Biological Psychiatry, 44(10), 1054-1059. https://doi.org/10.1016/S0006-3223(97)00475-7

Diest, I. V., Verstappen, K., Aubert, A. E., Widjaja, D., Vansteenwegen, D., \& Vlemincx, E. (2014). Inhalation/exhalation ratio modulates the effect of slow breathing on heart rate variability and relaxation. Applied Psychophysiology and Biofeedback, 39(3-4), 171-180. https://doi.org/10.1007/s10484-014-9253-x

Erkkilä, J., Punkanen, M., \& Fachner, J. (2012). Perspectives on creativity in improvisational, psychodynamic music therapy. In D. Hargreaves, D. Miell, \& R. MacDonald (Eds.), Musical imaginations: Multidisciplinary perspectives on creativity, performance and perception (pp. 414-428). Oxford: Oxford University Press.

Erkkilä, J., Punkanen, M., Fachner, J., Ala-Ruona, E., Pöntiö, I., Tervaniemi, M., ... Gold, C. (2011). Individual music therapy for depression: Randomised controlled trial. The British Journal of Psychiatry, 199(2), 132-139. https://doi.org/10.1192/bjp.bp.110.085431

Flick, U. (2004). Triangulation in qualitative research. In U. Flick, E. von Kardoff, \& I. Steinke (Eds.), A companion to qualitative research (pp. 178-183). London, Thousand Oaks, New Dehli: SAGE Publications.

Gevirtz, R. (2013). The promise of heart rate variability biofeedback: Evidence-based applications. Biofeedback, 41(3), 110.

Greenberg, L. S., \& Pascual-Leone, A. (2006). Emotion in psychotherapy: A practice-friendly research review. Journal of Clinical Psychology, 62(5), 611-630. https://doi.org/10.1002/jclp.20252

Grocke, D., \& Wigram, T. (2007). Receptive mehtods and relaxation for adults. In Receptive methods in music therapy: Techniques and clinical applications for music therapy clinicians, educators and students (1st ed., pp. 89-126). London: Jessica Kingsley Publishers.

Hayes, A. M., Beevers, C. G., Feldman, G. C., Laurenceau, J.-P., \& Perlman, C. (2005). Avoidance and processing as predictors of symptom change and positive growth in an integrative therapy for depression. International Journal of Behavioral Medicine, 12(2), 111-122. https://doi.org/10.1207/s15327558ijbm1202_9

Heathers, J. A. J. (2014). Everything Hertz: Methodological issues in short-term frequency-domain 
HRV. Cardiac Electrophysiology, 5, 177. https://doi.org/10.3389/fphys.2014.00177

Hunt, M. G. (1998). The only way out is through: Emotional processing and recovery after a depressing life event. Behaviour Research and Therapy, 36(4), 361-384. https://doi.org/10.1016/S0005-7967(98)00017-5

Jerath, R., Edry, J. W., Barnes, V. A., \& Jerath, V. (2006). Physiology of long pranayamic breathing: Neural respiratory elements may provide a mechanism that explains how slow deep breathing shifts the autonomic nervous system. Medical Hypotheses, 67(3), 566-571. https://doi.org/10.1016/j.mehy.2006.02.042

Karavidas, M. K., Lehrer, P. M., Vaschillo, E., Vaschillo, B., Marin, H., Buyske, S., ... Hassett, A. (2007). Preliminary results of an open label study of heart rate variability biofeedback for the treatment of major depression. Applied Psychophysiology and Biofeedback, 32(1), 19-30. https://doi.org/10.1007/s10484-006-9029-z

Krygier, J. R., Heathers, J. A. J., Shahrestani, S., Abbott, M., Gross, J. J., \& Kemp, A. H. (2013). Mindfulness meditation, well-being, and heart rate variability: A preliminary investigation into the impact of intensive Vipassana meditation. International Journal of Psychophysiology, 89(3), 305-313. https://doi.org/10.1016/j.ijpsycho.2013.06.017

Lehrer, P. M. (2007). Biofeedback training to increase heart rate variability. In P. M. Lehrer, R. L. Woolfolk, \& W. E. Sime (Eds.), Principles and practice of stress management (3rd ed., pp. 227-248). New York, London: Guilford Press.

Lehrer, P. M., \& Gevirtz, R. (2014). Heart rate variability biofeedback: How and why does it work? Frontiers in Psychology, 5. https://doi.org/10.3389/fpsyg.2014.00756

MacDonald, R. A., \& Wilson, G. B. (2014). Musical improvisation and health: A review. Psychology of Well-Being, 4(1), 20. https://doi.org/10.1186/s13612-014-0020-9

Magagnin, V., Bassani, T., Bari, V., Turiel, M., Maestri, R., Pinna, G. D., \& Porta, A. (2011). Nonstationarities significantly distort short-term spectral, symbolic and entropy heart rate variability indices. Physiological Measurement, 32(11), 1775. https://doi.org/10.1088/09673334/32/11/S05

McAleavey, A. A., \& Castonguay, L. G. (2015). The process of change in psychotherapy: Common and unique factors. In O. C. G. Gelo, A. Pritz, \& B. Rieken (Eds.), Psychotherapy Research (pp. 293-310). Vienna: Springer. https://doi.org/10.1007/978-3-7091-1382-0_20

McCraty, R., \& Zayas, M. A. (2014). Cardiac coherence, self-regulation, autonomic stability, and psychosocial well-being. Frontiers in Psychology, 5.

https://doi.org/10.3389/fpsyg.2014.01090

Mergenthaler, E. (1996). Emotion-abstraction patterns in verbatim protocols: A new way of describing psychotherapeutic processes. Journal of Consulting and Clinical Psychology, 64(6), 1306-1315. https://doi.org/10.1037/0022-006X.64.6.1306

Mergenthaler, E. (2008). Resonating minds: A school-independent theoretical conception and its empirical application to psychotherapeutic processes. Psychotherapy Research, 18(2), 109126. https://doi.org/10.1080/10503300701883741

Mergenthaler, E., \& Stinson, C. (1992). Psychotherapy transcription standards. Psychotherapy Research, 2(2), 125-142. https://doi.org/10.1080/10503309212331332904

Pagnini, F., Manzoni, G. M., Castelnuovo, G., \& Molinari, E. (2013). A brief literature review about relaxation therapy and anxiety. Body, Movement and Dance in Psychotherapy, 8(2), 71-81. https://doi.org/10.1080/17432979.2012.750248

Pal, G. K., Velkumary, S., \& Madanmohan, null. (2004). Effect of short-term practice of breathing exercises on autonomic functions in normal human volunteers. The Indian Journal of Medical Research, 120(2), 115-121.

Pellitteri, J. (2009). Emotional processes in music therapy. Gilsum, NH: Barcelona Publishers.

Pos, A. E., Greenberg, L. S., \& Warwar, S. H. (2009). Testing a model of change in the experiential treatment of depression. Journal of Consulting and Clinical Psychology, 77(6), 1055-1066. https://doi.org/http://dx.doi.org/10.1037/a0017059

Prensner, J. D., Yowler, C. J., Smith, L. F., Steele, A. L., \& Fratianne, R. B. (2001). Music therapy 
for assistance with pain and anxiety management in burn treatment. The Journal of Burn Care \& Rehabilitation, 22(1), 83-88-83.

Raymond, J., Sajid, I., Parkinson, L. A., \& Gruzelier, J. H. (2005). Biofeedback and dance performance: A preliminary investigation. Applied Psychophysiology and Biofeedback, 30(1), 65-73. https://doi.org/10.1007/s10484-005-2175-x

Reyes del Paso, G. A., Langewitz, W., Mulder, L. J. M., van Roon, A., \& Duschek, S. (2013). The utility of low frequency heart rate variability as an index of sympathetic cardiac tone: A review with emphasis on a reanalysis of previous studies. Psychophysiology, 50(5), 477-487. https://doi.org/10.1111/psyp.12027

Rivecourt, M. D., Kuperus, M. N., Post, W. J., \& Mulder, L. J. M. (2008). Cardiovascular and eye activity measures as indices for momentary changes in mental effort during simulated flight. Ergonomics, 51(9), 1295-1319. https://doi.org/10.1080/00140130802120267

Salahuddin, L., Cho, J., Jeong, M. G., \& Kim, D. (2007). Ultra short term analysis of heart rate variability for monitoring mental stress in mobile settings. In 29th Annual International Conference of the IEEE Engineering in Medicine and Biology Society, 2007. EMBS 2007 (pp. 4656-4659). https://doi.org/10.1109/IEMBS.2007.4353378

Sandercock, G. R. H., Bromley, P. D., \& Brodie, D. A. (2005). The reliability of short-term measurements of heart rate variability. International Journal of Cardiology, 103(3), 238247. https://doi.org/10.1016/j.ijcard.2004.09.013

Schwarz, A. M., Schächinger, H., Adler, R. H., \& Goetz, S. M. (2003). Hopelessness is associated with decreased heart rate variability during championship chess games. Psychosomatic Medicine, 65(4), 658-661. https://doi.org/10.1097/01.PSY.0000075975.90979.2A

Shaffer, F., McCraty, R., \& Zerr, C. L. (2014). A healthy heart is not a metronome: An integrative review of the heart's anatomy and heart rate variability. Frontiers in Psychology, 5. https://doi.org/10.3389/fpsyg.2014.01040

Singer, J. L. (1974). Imagery and daydream methods in psychotherapy and behaviour modification. New York: Academic Press Inc.

Sloan, D. M., \& Kring, A. M. (2007). Measuring changes in emotion during psychotherapy: Conceptual and methodological issues. Clinical Psychology: Science and Practice, 14(4), 307-322. https://doi.org/10.1111/j.1468-2850.2007.00092.x

Smith, A.-L., Owen, H., \& Reynolds, K. J. (2013). Heart rate variability indices for very short-term (30 beat) analysis. Part 2: validation. Journal of Clinical Monitoring and Computing, 27(5), 577-585. https://doi.org/10.1007/s10877-013-9473-2

Smith, J. C., Wedell, A. B., Kolotylo, C. J., Lewis, J. E., Byers, K. Y., \& Segin, C. M. (2000). ABC relaxation theory and the factor structure of relaxation states, recalled relaxation activities, dispositions, and motivations. Psychological Reports, 86(3 Pt 2), 1201-1208. https://doi.org/10.2466/pr0.2000.86.3c.1201

Strauss-Blasche, G., Moser, M., Voica, M., McLeod, D., Klammer, N., \& Marktl, W. (2000). Relative timing of inspiration and expiration affects respiratory sinus arrhythmia. Clinical and Experimental Pharmacology and Physiology, 27(8), 601-606. https://doi.org/10.1046/j.1440-1681.2000.03306.x

Sutarto, A. P., Wahab, M. N. A., \& Zin, N. M. (2012). Resonant breathing biofeedback training for stress reduction among manufacturing operators. International Journal of Occupational Safety and Ergonomics, 18(4), 549-561. https://doi.org/10.1080/10803548.2012.11076959

Tarvainen, M. P., Niskanen, J.-P., Lipponen, J. A., Ranta-aho, P. O., \& Karjalainen, P. A. (2014). Kubios HRV - Heart rate variability analysis software. Computer Methods and Programs in Biomedicine, 113(1), 210-220. https://doi.org/10.1016/j.cmpb.2013.07.024

Tarvainen, M. P., Ranta-aho, P. O., \& Karjalainen, P. A. (2002). An advanced detrending method with application to HRV analysis. IEEE Transactions on Biomedical Engineering, 49(2), 172-175. https://doi.org/10.1109/10.979357

Task Force of the European Society of Cardiology and the North American Society of Pacing and Electrophysiology. (1996). Heart rate variability standards of measurement, physiological 
interpretation, and clinical use. Circulation, 93(5), 1043-1065.

https://doi.org/10.1161/01.CIR.93.5.1043

Thayer, J. F., Yamamoto, S. S., \& Brosschot, J. F. (2010). The relationship of autonomic imbalance, heart rate variability and cardiovascular disease risk factors. International Journal of Cardiology, 141(2), 122-131. https://doi.org/10.1016/j.ijcard.2009.09.543

Vaschillo, E., Lehrer, P., Rishe, N., \& Konstantinov, M. (2002). Heart rate variability biofeedback as a method for assessing baroreflex function: A preliminary study of resonance in the cardiovascular system. Applied Psychophysiology and Biofeedback, 27(1), 1-27. https://doi.org/10.1023/A:1014587304314

Watson, D., Clark, L. A., \& Tellegen, A. (1988). Development and validation of brief measures of positive and negative affect: The PANAS scales. Journal of Personality and Social Psychology, 54(6), 1063-1070.

Weippert, M., Kumar, M., Kreuzfeld, S., Arndt, D., Rieger, A., \& Stoll, R. (2010). Comparison of three mobile devices for measuring R-R intervals and heart rate variability: Polar S810i, Suunto t6 and an ambulatory ECG system. European Journal of Applied Physiology, 109(4), 779-786. https://doi.org/10.1007/s00421-010-1415-9

Winkelman, M. (2010). Shamanism: A biopsychosocial paradigm of consciousness and healing (2nd ed.). Santa Barbara, CA: Praeger (ABC-CLIO).

Wu, S.-D., \& Lo, P.-C. (2008). Inward-attention meditation increases parasympathetic activity: A study based on heart rate variability. Biomedical Research, 29(5), 245-250. https://doi.org/10.2220/biomedres.29.245

Zucker, T. L., Samuelson, K. W., Muench, F., Greenberg, M. A., \& Gevirtz, R. N. (2009). The effects of respiratory sinus arrhythmia biofeedback on heart rate variability and posttraumatic stress disorder symptoms: A pilot study. Applied Psychophysiology and Biofeedback, 34(2), 135-143. https://doi.org/10.1007/s10484-009-9085-2 